\title{
Antibiotics in biliary disease: the relative importance of antibiotic concentrations in the bile and serum
}

\author{
M. R. B. KEIGHLEY, R. B. DRYSDALE, A. H. QUORAISHI, D. W. BURDON, \\ AND J. ALEXANDER-WILLIAMS ${ }^{1}$
}

From the General Hospital and University of Birmingham, Birmingham

SUMMARY A randomised controlled trial has been performed in 150 patients undergoing biliary operations to determine whether an antibiotic which is excreted almost entirely in bile (rifamide) is to be preferred to one having satisfactory serum levels only (gentamicin). Patients were allocated to one of three groups: 50 received gentamicin, 50 received rifamide, and there were 50 controls who received no antibiotic cover. In the absence of duct obstruction, rifamide achieved extremely high bile levels but low serum concentrations. However, in jaundiced patients, both the bile and the serum concentrations of rifamide were too low to be of therapeutic value. The incidence of postoperative sepsis was not reduced by rifamide compared with controls. In contrast, gentamicin achieved adequate serum concentrations in $88 \%$ of patients. Despite poor bile levels, gentamicin was associated with a significant reduction of wound infection from $22 \%$ to $6 \%$ and septicaemia from $14 \%$ to $2 \%$ compared with controls. To reduce the septic complications of biliary operations, adequate serum levels of an effective antimicrobial are more important than an antibiotic, which is excreted almost entirely into the bile.

Organisms can be isolated from the bile in approximately a third of patients having operations on the biliary tract (Edlund et al., 1958). If the bile duct contains stones and the patient is jaundiced at operation, bacteria are present in $90 \%$ of cases (Flemma et al., 1967). After biliary operations, most of the clinical infections in the wound and in the bloodstream are associated with the presence of bacteria in the bile (Mason, 1968; Keighley et al., 1974). Therefore, it is those patients with calculous obstructive jaundice who are most likely to develop wound sepsis or septicaemia after elective operation.

Prophylactic antibiotics have been shown to minimise the incidence of sepsis after biliary surgery (Bevan and Williams, 1971), and it has been suggested that an antibiotic that achieves high concentrations in the bile should be used (Acocella et al., 1968). Concentrations of antibiotic in the bile in patients having operations for biliary disease are inadequate if the biliary tract is obstructed, even if the antibiotic is normally excreted in high concentrations into the bile (Zaslow et al., 1947; Zaslow et al., 1950; Zaslow and Rosenthal, 1954; Khan and Scott, 1967; Mortimer et al., 1969; Sales et al., 1972).

${ }^{1}$ Reprint requests to Mr J. Alexander-Williams.

Received for publication 7 April 1976
The purpose of this study is to compare the results of prophylaxis with an antibiotic almost entirely excreted in the bile (rifamide) with one having adequate concentrations only in the serum (gentamicin).

\section{Methods}

\section{CLINICAL STUDY}

One hundred and fifty patients undergoing operations on the biliary tract were allocated at random to one of three groups. Fifty received gentamicin, 50 were given rifamide, and there were 50 control patients who received no antibiotic cover. Randomisation was stratified to provide equal numbers of jaundiced patients in each group (Table 1). Instructions were enclosed in a sealed envelope that was opened by the house officer on the evening before operation. Patients in both treated groups received their first intramuscular injection at 6 a.m. on the day of operation and a second dose in the anaesthetic room. After operation, the antibiotics were continued by the intramuscular route eight hourly for five days. The doses of gentamicin and rifamide were $80 \mathrm{mg}$ and $150 \mathrm{mg}$ respectively.

During the operation, bile and serum samples were collected simultaneously for antibiotic assay 
Table 1 Comparison of groups

\begin{tabular}{lrrr}
\hline $\begin{array}{l}\text { Factors which predispose } \\
\text { to presence of bacteria } \\
\text { in bile }\end{array}$ & Gentamicin 50 & Controls 50 & Rifamide 50 \\
\hline Age over 70 yr & 8 & 7 & 6 \\
Jaundice at operation & 11 & 11 & 11 \\
$\quad$ Stones & 7 & 7 & 8 \\
$\quad$ Carcinoma & 4 & 4 & 3 \\
Emergency operation & 3 & 3 & 4 \\
Exploration of common & 16 & 16 & 13 \\
bile duct & 12 & 12 & 13 \\
$\quad$ Stones found & 12 & 28 & 28 \\
Drainage of subhepatic & 29 & & \\
\hline
\end{tabular}

*Determined by design of the trial (see text).

immediately before operative cholangiography. Bile was aspirated from the gall bladder in 78 patients receiving antibiotics $(78 \%)$ and from the bile duct through the cholangiogram catheter in 70 of these cases $(70 \%)$. Wound sepsis was defined as the presence of pus in the incision. The wounds were examined by one independent observer who did not know whether or not the patient was receiving antibiotics. Blood culture samples were taken when the patient returned to the ward after operation and on the third, fifth, and seventh postoperative days. Blood cultures were repeated after the ' $T$ ' tube cholangiogram and in any patient with an unexplained pyrexia.

Four patients (three of whom were jaundiced) were withdrawn from the trial because the antibiotic policy was changed within 72 hours of operation. To keep the total number of patients in the groups equal, replacement randomisation cards were used. For this reason, the groups were well matched with respect to the number of patients over 70 years, emergency operation, the incidence of ductal exploration, and the use of drains (Table 1).

\section{MICROBIOLOGICAL STUDY}

Bile for culture was taken to the laboratory promptly. It was cultured aerobically on blood agar and MacConkey agar and anaerobically with $10 \% \mathrm{CO}_{2}$ on blood agar and lysed blood agar containing neomycin or kanamycin and menadione. Cultures were incubated at $37^{\circ} \mathrm{C}$ for 48 hours. Bacteria were identified by their cultural properties, colonial appearance, and biochemical reactions. Viable counts were not measured, though previous results from this unit have shown that over $90 \%$ of positive bile cultures contain more than $10^{5}$ organisms per ml (Keighley et al., 1975). The method of bile cultures was the same in the treated groups and the controls. It is possible that preoperative antibiotics may have influenced the incidence of bacterbilia. However, the dilution produced by inoculating the bile on agar plates was sufficient to allow the growth of the most gentamicin-sensitive coliforms. Conversely, in patients receiving rifamide, the growth of bacteria from the bile may have been inhibited by the higher concentrations of rifamide in the bile.

The sensitivity of bacteria isolated from bile to gentamicin and rifamide was determined by measurement of the minimum inhibitory concentration (MIC) using the agar dilution method (Garrod et al., 1973). Serial dilutions of antibiotic were prepared from fresh solutions of rifamide and gentamicin.

Samples of bile and serum were stored at $-30^{\circ} \mathrm{C}$ for batch antibiotic assay. Gentamicin assays were performed on large plates measuring $32 \times 32 \mathrm{~cm}$ using Bacillus subtilis as the test organism. The method described by Khan and Scott (1967) was used for the rifamide assays. There was no appreciable difference in the sizes of the inhibition zones when rifamide and gentamicin were diluted with saline, serum, bile, or phosphate buffer ( $\mathrm{pH} 7.0$ and $\mathrm{pH} \mathrm{8.0)}$. Therefore, in both assays, the standard dilutions were prepared in normal saline. A standard curve was constructed for each batch of assays by plotting the antibiotic concentration (log scale) against the diameter of the inhibition zone measured by calipers at two separate diameters. The antibiotic concentrations in the bile and serum were then calculated from the standard curves. Each estimation was performed in duplicate.

\section{Results}

Bacteria were isolated from the bile in 19 of the untreated control patients (38\%) compared with 12 patients receiving gentamicin $(24 \%)$ and 10 cases receiving rifamide $(20 \%)$. The differences are not statistically significant. Nevertheless, Escherichia coli was isolated less frequently from both treated groups than from the controls (Table 2). In patients with jaundice, the incidence of organisms in the bile was approximately the same in all three groups.

The MICs of gentamicin and rifamide are shown in Table 3. Eighty-two per cent of organisms isolated from the bile were inhibited by $2.0 \mathrm{mg} / 1$ gentamicin. The organisms resistant to this concentration included all of the anaerobic bacteria and many faecal streptococci. Eighty-four per cent of biliary micro-organisms were inhibited by $31 \mathrm{mg} / 1$ rifamide; those that were not, included some of the aerobic Gram negative bacilli (Klebsiella aerogenes, Escherichia coli, Proteus spp., and Pseudomonas aeruginosa).

The inhibitory concentrations of gentamicin and rifamide were used to define what was considered to be an 'adequate' concentration of antibiotic in patients with biliary disease. The level chosen as 
Table 2 Organisms cultured from bile at operation

\begin{tabular}{llcl}
\hline Organism & Gentamicin 50 & Controls 50 & Rifamide 50 \\
\hline Escherichia coli & 4 & 14 & 5 \\
Klebsiella aerogenes & 3 & 5 & 1 \\
Streptococcus faecalis & 4 & 4 & 3 \\
Streptococcus viridans & 2 & - & 1 \\
Proteus spp. & 1 & 1 & 1 \\
Enterobacter cloacae & 1 & 1 & 1 \\
Staphylococcus albus & - & 1 & 1 \\
Pseudomonas aeruginosa & - & - & 1 \\
Acinetobacter anitratus & - & - & 1 \\
Enterobacter aerogenes & 1 & - & - \\
Serratia sp. & - & 1 & - \\
Anaerobic streptococci & 1 & - & - \\
Bacteroides fragilis & - & 1 & - \\
Clostridium welchii & - & 3 & - \\
Total number of patients & & & \\
with microorganisms in & & $19(38 \%)$ & $10(20 \%)$ \\
bile & $12(24 \%)$ & 7 & 6 \\
Jaundiced patients & 5 & 12 & 4 \\
Not jaundiced & 7 & &
\end{tabular}

'adequate' was twice the MIC for over $80 \%$ of biliary isolates - that is, $4 \mathrm{mg} / \mathrm{l}$ gentamicin and $62 \mathrm{mg} / \mathrm{l}$ rifamide). To justify this concept of an 'adequate' antibiotic concentration, it is important to know that the levels in the bile and serum at operation were peak levels. Peak serum concentrations of gentamicin usually occur one hour after intramuscular injection (Reeves, 1974). To measure peak bile levels of rifamide and gentamicin, samples were collected hourly from eight patients with ' $T$ ' tubes. The highest levels of both antibiotic were found one hour after intramuscular administration. The mean time interval between the immediate preoperative injection and the collection of the bile and serum samples for assay was 50.3 minutes (range: $30-65$ minutes).

The results of the antibiotic assays are shown in Figs. 1 and 2. The plot above the horizontal line represents 'adequate' concentrations of antibiotic. 'Adequate' serum gentamicin concentrations were
Table 4 Results of prophylactic therapy

\begin{tabular}{llll}
\hline Septic complications & Gentamicin 50 & Controls 50 & Rifamide 50 \\
\hline Wound sepsis & $3^{*}$ & 11 & 5 \\
Jaundiced & 1 & 5 & 3 \\
Not jaundiced & 2 & 6 & 2 \\
& & & \\
Bacteraemia & $1^{*}(0)$ & $7(5)$ & $4(4)$ \\
Jaundiced & 0 & 3 & 3 \\
Not jaundiced & 1 & 4 & 1 \\
\hline
\end{tabular}

*Indicates that the difference between the treated groups and controls was significant $(P<0.05)$.

Figures in parentheses refer to numbers of patients with clinical septicaemia.

present in 37 of 43 patients ( $88 \%$ ) but the levels in bile were extremely low. Only two gall bladder samples and none of the collections from the bile duct exceeded $4 \mathrm{mg} / \mathrm{l}$. In contrast, none of the patients had 'adequate' serum rifamide concentrations. Nevertheless, provided that there was no biliary obstruction, rifamide bile levels were extremely high, in many instances exceeding $1000 \mathrm{mg} / \mathrm{l}$. However, when the cystic duct was obstructed, levels in the gall bladder were less than $5 \mathrm{mg} / 1$ and 'adequate' bile levels were achieved in only two of the jaundiced patients.

The influence of prophylactic antibiotic therapy on the incidence of postoperative sepsis is shown in Table 4. Wound sepsis occurred in 11 controls $(22 \%)$, in five receiving rifamide $(10 \%)$, and in only three of the patients who were given gentamicin $(6 \%)$. The rate of wound infection in patients receiving gentamicin was significantly lower than in the control group ( $P<0.05)$. Bacteraemia occurred in seven of the control patients; five had clinical evidence of septicaemia and three were jaundiced. In the group receiving rifamide there were four patients with positive blood cultures: all had rigors and three

Table 3 Minimum inhibitory concentrations of gentamicin and rifamide

\begin{tabular}{|c|c|c|c|c|c|c|c|c|c|c|c|c|c|c|c|c|c|}
\hline & \multicolumn{9}{|c|}{ Gentamicin $(m g / l)$} & \multicolumn{8}{|c|}{ Rifamide $(\mathrm{mg} / \mathrm{l})$} \\
\hline & 0.06 & $0 \cdot 125$ & $0 \cdot 25$ & $0 \cdot 5$ & $1 \cdot 0$ & $2 \cdot 0$ & $4 \cdot 0$ & $8 \cdot 0$ & $>8 \cdot 0$ & $1 \cdot 9$ & $3 \cdot 9$ & $7 \cdot 8$ & $15 \cdot 6$ & $31 \cdot 2$ & $62 \cdot 5$ & 125 & $>125$ \\
\hline Klebsiella aerogenes & & & 5 & 5 & & & & & & & & 1 & 4 & 2 & 1 & 1 & \\
\hline Streptococcus faecalis & & & & & & 3 & 2 & 4 & 2 & 4 & 2 & 2 & 1 & 2 & & & \\
\hline Streptococcus viridans & & & & & 2 & 1 & & & & & 1 & 2 & & & & & \\
\hline Anaerobic streptococci & & & & & & 1 & & & & & & & & & & & \\
\hline Pseudomonas aeruginosa & & & & & 1 & & & & & & & & & & 1 & & \\
\hline $\begin{array}{l}\text { Acinetobacter anitratus } \\
\text { Clostridium welchii }\end{array}$ & & & & 1 & & 1 & & & & & & & & 1 & & & \\
\hline Bacteroides sp. & & & & & & & & & 1 & 1 & & & & & & & \\
\hline Enterobacter cloacae & & & 2 & 1 & & & & & & 1 & & & 2 & & & & \\
\hline Serratia sp. & & & & 1 & & & & & & & & & 1 & & & & \\
\hline Staphylococcus albus & & & 2 & & & & & & & 2 & & & & & & & \\
\hline Total & & 1 & 11 & 19 & 13 & 8 & 4 & 4 & 3 & 8 & 3 & 7 & 19 & 11 & 3 & 4 & 2 \\
\hline
\end{tabular}




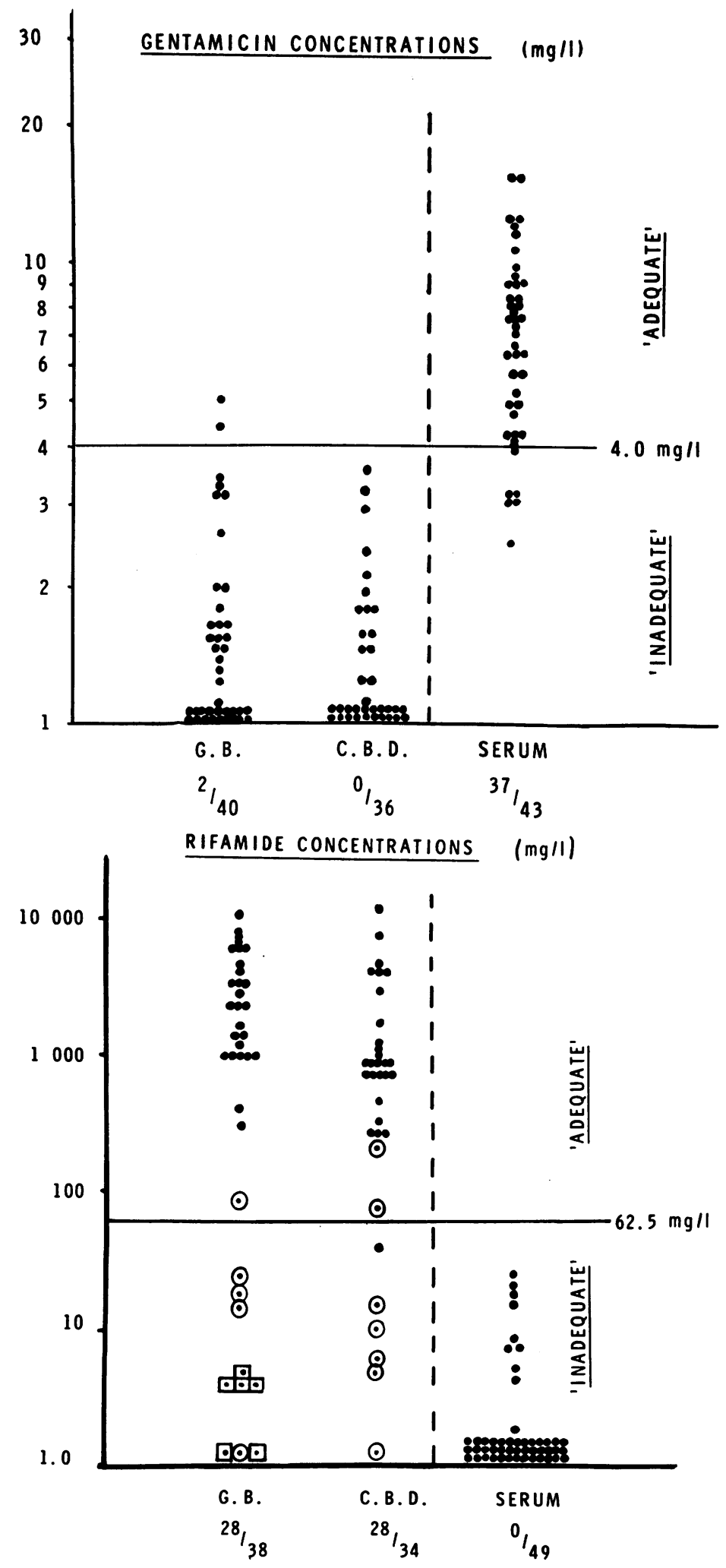

Fig. 1 Gentamicin assay in bile and serum at operation.

Fig. 2 Rifamide assay in bile and serum at operation. $\odot=$ Bile from jaundiced patients. $\square=G . B$. bile in patients with cystic duct occlusion. 
were jaundiced. In contrast, only one patient receiving gentamicin developed bacteraemia: this occurred in a patient who was not jaundiced and it was not associated with clinical signs of septicaemia. The incidence of bacteraemia was significantly lower in the gentamicin group than in patients who received no antibiotic cover.

\section{Discussion}

These results indicate that antibiotics which are present only in the serum in adequate concentrations are more likely to be effective as prophylaxis in biliary surgery than ones which are excreted almost entirely into the bile. Even if an antibiotic is excreted through the liver, biliary concentrations are rarely sufficient to be effective in the presence of biliary obstruction. The group of patients who are most likely to have an infected biliary tract at elective operation, and who therefore may develop septicaemia, are those with calculous obstructive jaundice (Maddocks et al., 1973).

In patients who were receiving no antibiotic cover, organisms were isolated in the bile from $73 \%$ of the jaundiced patients but in only $33 \%$ of those without jaundice. In the control patients who were jaundiced, the incidence of wound infection was $45 \%$ and bacteraemia was $27 \%$. Complications were much less frequent in the controls who were not jaundiced (wound sepsis and bacteraemia $15 \%$ and $10 \%$ respectively). The influence of antibiotics on postoperative sepsis in the non-icteric patients was the same in both of the treated groups even though gentamicin was not excreted in adequate quantities into the bile. Furthermore, in the jaundiced patients rifamide failed to achieve adequate bile levels. The incidence of bacterbilia and postoperative sepsis in the jaundiced patients receiving rifamide was the same as in the controls. However, even in the jaundiced patients, gentamicin reduced wound sepsis and bacteraemia did not occur.

Rifamide was chosen for this study principally because we wished to compare gentamicin with an antibiotic known to give high concentrations in the bile rather than in the serum. Khan and Scott (1967) have demonstrated that serum levels of rifamide, even in jaundiced patients, rarely exceed the MIC of most Gram-positive organisms and are far too low to be of influence against the 'coliforms'. Rifampicin is more active than rifamide (Mitchison, 1970). Rifampicin is, however, available only as an oral preparation and is therefore unsuitable for use in patients undergoing operations that may be associated with a period of postoperative ileus. Furthermore, rifampicin, unlike rifamide, achieves satisfactory blood levels, and we were anxious to investigate an antibiotic which depended solely upon biliary concentrations for effective prophylaxis.

Even though there was a significant reduction in morbidity when gentamicin was given to patients undergoing operations on the biliary tract, we would not advise its use in prophylaxis for all patients because of the dangers of nephrotoxicity and damage to the VIII nerve. The dose of gentamicin was inadequate for some of the patients and, for this reason, we would now advise that $5 \mathrm{mg} / \mathrm{kg}$ body weight should be given daily in divided doses. The results of this study have shown that gentamicin reduced the incidence of postoperative infection only when the bile contained organisms at the time of operation (see Table 5). We therefore advise that prophylactic chemotherapy should be given only when the bile contains bacteria. Methods of identifying patients with bacterbilia during and before operation have been studied (Engstrom et al., 1971; Keighley et al., 1976) so that the indiscriminant use of prophylactic antibiotics can be avoided. Nevertheless, in patients requiring emergency operation, for surgical relief of

Table 5 Incidence of wound sepsis and bacteraemia related to presence of absence of bacteria in bile at operation

\begin{tabular}{lccc}
\hline & Gentamicin & Controls & Rifamide \\
\hline No organisms in bile & 38 & 31 & 40 \\
Wound sepsis & 2 & 2 & 1 \\
Bacteraemia & 0 & 0 & 0 \\
Bacteria in bile & 12 & 19 & 10 \\
Wound sepsis & $1^{*}$ & 9 & 4 \\
Bacteraemia & 1 & 7 & 4 \\
\hline
\end{tabular}

*Indicates that the difference between the treated group and the controls was significant $(P<0.05)$.

obstructive jaundice and in patients with cholangitis where antibiotics are indicated, high serum levels of an effective antibiotic are more likely to be of value than an antibiotic which is almost entirely excreted into the bile.

We wish to thank Mr R. M. Baddeley, Mr J. A. C. Edwards, Mr G. D. Oates, and Mr G. T. Watts for allowing us to study patients under their care and Miss V. A. Price for typing the manuscript.

\section{References}

Acocella, G., Matiussi, R., Nicolis, F. B., Pallanza, R., and Tenconi, L. T. (1968). Biliary excretion of antibiotics in man. Gut, 9, 536-545.

Bevan, P. G., and Williams, J. D. (1971). Rifamide in acute cholecystitis and biliary surgery. British Medical Journal, 3, 284-287.

Edlund, Y. A., Mollstedt, B. O., and Ouchterlony, O. (1959). Bacteriological investigations of the biliary system and liver in biliary tract disease correlated to clinical data and 
microstructure of the gallbladder and liver. Acta Chirurgica Scandinavica, 116, 461-476.

Engström, J., Hellström, K., Hogman, L., and Lönnqvist, B. (1971). Microorganisms of the liver, biliary tract, and duodenal aspirates in biliary diseases. Scandinavian Journal of Gastroenterology, 6, 177-182.

Flemma, R. J., Flint, L. M., Osterhout, S., and Shingleton, W. W. (1967). Bacteriologic studies of biliary tract infection. Annals of Surgery, 166, 563-572.

Garrod, L. P., Lambert, H. P., and O'Grady, F. (1973). Antibiotic and Chemotherapy. 4th edn., p. 509. Churchill Livingstone: Edinburgh.

Keighley, M. R. B., Baddeley, R. M., Burdon, D. W., Edwards, J. A. C., Quoraishi, A. H., Oates, G. D., Watts, G. T., and Alexander-Williams, J. (1975). A controlled trial of parenteral prophylactic gentamicin therapy in biliary surgery. British Journal of Surgery, 62, 275-279.

Keighley, M. R. B., Crapp, A. R., Flinn, R., and AlexanderWilliams, J. (1976). Multivariate analysis of clinical and operative findings associated with biliary sepsis. British Journal of Surgery (In press).

Keighley, M. R. B., Lister, D. M., Jacobs, S. I., and Giles, G. R. (1974). Hazards of surgical treatment due to microorganisms in the bile. Surgery, 75, 578-583.

Khan, G. A., and Scott, A. J. (1967). The place of rifamide-
B-diethylamide in the treatment of cholangitis complicating biliary obstruction. British Journal of Pharmacology, 31, 506-512.

Maddocks, A. C., Hilson, G. R. F., and Taylor, R. (1973). The bacteriology of the obstructed biliary tree. Annals of the Royal College of Surgeons, 52, 316-319.

Mason, G. R. (1968). Bacteriology and antibiotic selection in biliary surgery. Archives of Surgery, 97, 533-537.

Mitchison, D. A. (1970). The rifamycins and rifampicin. Prescribers' Journal, 10, 83-86.

Mortimer, P. R., Mackie, D. B., and Haynes, S. (1969). Ampicillin levels in human bile in the presence of biliary tract disease. British Medical Journal, 3, 88-89.

Reeves, D. S. (1974). Gentamicin therapy. British Journal of Hospital Medicine, 12, 837-850.

Zaslow, J., Counseller, V. S., and Heilman, F. R. (1947). The excretion and concentration of penicillin and streptomycin in abnormal human biliary tract, pts. 1 and 2 . Surgery, Gynecology, and Obstetrics, 84, 16-20, 140-152.

Zaslow, J., Hewlett, T. H., and Lorry, R. W. (1950). The excretion and concentration of aureomycin in the abnormal human biliary tract. Gastroenterology, 16, 475-483.

Zaslow, J., and Rosenthal, A. (1954). The excretion and concentration of terramycin in abnormal human biliary tract. Annals of Surgery, 139, 478-483. 